\title{
¿Cómo evalúas a tus alumnos de instrumento? Ideas del profesorado de conservatorio acerca de los procedimientos de evaluación
}

\author{
Aránzazu GONZÁLEZ ROYO ${ }^{1}$, Alfredo BAUTISTA ${ }^{2}$ \\ (1) C.I.E.M. Federico Moreno Torroba (Madrid, España) \\ ${ }^{(2)}$ Nanyang Technological University, National Institute of Education (Singapur)
}

(Recibido, 25 septiembre 2017; Aceptado, 2 enero 2018)

\begin{abstract}
RESUMEN: En el campo de las enseñanzas de instrumento, existen estudios que analizan los procedimientos que los profesores utilizan para evaluar en contextos de evaluación procesual, así como la fiabilidad y validez de los procedimientos de calificación en contextos de evaluación final. Sin embargo, existen pocos estudios centrados en analizar las ideas que tienen los profesores sobre cómo evaluar durante todo el ciclo de evaluación continua (evaluación procesual y final). El objetivo de este estudio exploratorio fue investigar las ideas del profesorado de conservatorios sobre los procedimientos de evaluación. Concretamente, analizamos las ideas relativas a la forma de evaluar a los alumnos clase a clase y en los exámenes, así como el grado de acuerdo percibido por los profesores con la manera de evaluar de sus compañeros de departamento. Se realizó una entrevista semi-estucturada a 18 profesores de instrumento de la Comunidad de Madrid (España). Las entrevistas se analizaron mediante análisis del contenido, de acuerdo a cuatro dimensiones: cómo se evalúa, qué se evalúa, qué referente se utiliza y para qué se evalúa. Los resultados mostraron que, pese a que las ideas relativas a los contextos de evaluación procesual y final difieren sustancialmente, ambas tienden a ser superficiales respecto a la forma de evaluar y fomentar los aprendizajes del alumno. Asimismo, los profesores manifestaron no estar de acuerdo con la forma en que evalúan sus compañeros de departamento. Sugerimos la necesidad de que los departamentos de instrumento se esfuercen en explicitar y consensuar los procedimientos de evaluación.
\end{abstract}

Palabras clave: Profesores de instrumento, conservatorio, procedimientos de evaluación, evaluación continua, pensamiento del profesor.

\section{How do you assess your instrumental students? Conservatory teachers' ideas about assessment procedures}

\begin{abstract}
In the field of instrumental teaching, research studies have analyzed the procedures used by teachers to assess students within ongoing assessment settings, as well as the reliability and validity of procedures used in final assessment settings. However, few studies have specifically focused on analyzing teachers' ideas on how to assess throughout all the continuous assessment cycle (process and final assessment). The aim of this exploratory study was to describe the ideas held by instrumental teachers from music conservatories regarding assessment procedures. In particular, we analyzed teachers' ideas on how to assess students in class and in exams, as well as their perceived degree of agreement with their fellow departmental colleagues. A semi-structured interview was conducted with
\end{abstract}


18 teachers from the Community of Madrid (Spain). The interviews were analyzed by content analysis, according to four dimensions: how to asses, what to assesses, what reference point is used, and what to assess for. Findings show that, although teachers' ideas about how to assess in class and in exams differ substantially, both tend to be superficial with regards to fostering student learning. Moreover, teachers expressed disagreement with the assessment procedures used by their departmental colleagues. We conclude that there is a need for instrumental departments to make explicit and reach agreements on assessment procedures.

Keywords: Music performance teachers, music conservatories, assessment procedures, continuous assessment, teacher thinking.

Correspondencia: Aránzazu González Royo. C.I.E.M. Federico Moreno Torroba. Calle Clara Campoamor, 10, 28025 Madrid. Email: aranzazugr@gmail.com.

\section{Introducción}

El proyecto curricular de corte constructivista que desarrolló la reforma educativa de la LOGSE (1990), y que se ha mantenido con la LOE (2006), planteó muchos desafíos para la educación musical (Pozo, Bautista, \& Torrado, 2008). En el ámbito de la evaluación se introdujo el concepto de evaluación 'continua', en contraposición al plan previo en el que la evaluación consistía únicamente en calificar en los exámenes finales (Decreto 2618, 1966). Este cambio implicó una modificación en la dimensión temporal de la evaluación, que pasó de ser un hecho puntual a ser una práctica inserta en el proceso de enseñanza-aprendizaje (Miras \& Solé, 1990). La evaluación continua incluye las fases de evaluación inicial, procesual y final (Casanova, 1995). El propósito fue que la evaluación atendiera a los procesos del aprendizaje, y no solamente se limitara a medir los resultados (Coll, Martín, \& Onrubia, 2001), en un proceso cíclico de toma de decisiones pedagógicas (Casanova, 1995).

La enseñanza de la interpretación instrumental presenta unas características particulares que nos permiten analizar la evaluación del proceso y del resultado del aprendizaje como un continuo pedagógico. Por una parte, las clases son principalmente individuales y el profesor de instrumento es el agente que regula y evalúa el proceso de enseñanza-aprendizaje, en ocasiones durante buena parte de la formación de los alumnos (si no toda). Además, los profesores como miembros del departamento de instrumento - deben concretan los procedimientos de evaluación, atendiendo a los objetivos y criterios de evaluación contenidos en el currículo, y adaptarlos a las necesidades de los alumnos de cada centro (Orden 1031, 2008).

Todo factor que impida al alumno tener una certeza sobre la forma en que va a ser

evaluado y/o calificado puede mermar su capacidad de previsión y, por lo tanto, de aprendizaje (Pozo, 1996). La evaluación tiene efectos retroactivos sobre el aprendizaje (Monereo, 2003). Una falta de coherencia dentro del ciclo evaluador se podría producir si, por ejemplo, los profesores evaluaran con unos procedimientos e intencionalidad pedagógica contradictorios en las distintas fases del ciclo evaluador. Ello también podría ocurrir en el caso de que el profesor exigiera del alumno la consecución de unos objetivos de aprendizaje diferentes a los trabajados durante las clases.

Hasta la fecha, existen pocos estudios realizados en España centrados en analizar las ideas que tienen los profesores de conservatorio sobre los procedimientos de evaluación en contextos de evaluación procesual y final, así como el grado en que los profesores están de 
acuerdo con sus compañeros de departamento en la forma en que evalúan. Estos son los objetivos generales del presente estudio.

\section{Los procedimientos de evaluación de las interpretaciones musicales de los alumnos en contextos académicos}

Coll y Remesal (2009) definen la evaluación como un procedimiento de recogida, análisis y valoración de la información obtenida en el proceso de enseñanza y aprendizaje. Principalmente, los procedimientos de evaluación estructuran y dan respuesta al ¿cómo se evalúa? Concretan la forma específica en que se lleva a cabo la evaluación de los alumnos (Ibarra \& Rodríguez, 2010), englobando todo el conjunto de acciones dirigidas al acto de evaluar (Pozo, 1996).

En la evaluación para el aprendizaje, consideramos la evaluación como un proceso integrado en los procesos de enseñanza-aprendizaje (Brown, 2004). La manera en que los profesores evalúan debería ser coherente con los resultados que pretendan obtener del aprendizaje, ya que cada procedimiento de evaluación promueve un tipo de aprendizaje determinado (Biggs, 1996). Consecuentemente, los procedimientos de evaluación se articulan con las mismas dimensiones con las que se produce la enseñanza y el aprendizaje. El ¿cómo se evalúa? está inevitablemente ligado a las dimensiones: ¿qué se evalúa?, ¿qué referente se utiliza? y ¿para qué se evalúa? (Bautista, Pérez Echeverría, \& Pozo, 2011; Zabalza, 1987).

Durante el proceso de aprendizaje del instrumento, el profesor promueve el desarrollo de las capacidades, conocimientos y destrezas musicales de los alumnos a través de diferentes estrategias didácticas (Pozo et al., 2008). Este proceso se lleva a cabo principalmente mediante el trabajo de un repertorio de obras de distintos estilos, con el que posteriormente se evalúa al alumno en escenarios de evaluación final. El repertorio de obras sería el medio fundamental que los profesores utilizan en las clases para desarrollar las capacidades del alumno (Bautista, Pérez Echeverría, \& Pozo, 2010; Torrado \& Pozo, 2008).

El profesor típicamente evalúa al alumno durante las clases estableciendo comparaciones entre lo que el alumno está tocando realmente, y cómo el mismo alumno toca - o querría que sonara lo que está tocando - (Hallam \& Creech, 2010). Por lo tanto, la retroalimentación que los alumnos reciben por parte del profesor debería promover el desarrollo de un "oído interno" por parte del alumno (Decreto 30, 2007, p. 8). El rol del profesor sería el de guía que ayuda al alumno a construir una representación artística de la partitura (Chaffin, Imreh, Lemieux, \& Chen, 2003), dirigiéndole en la regulación de los recursos cognitivos y motrices que favorezcan la producción de esa imagen o representación sonora (interpretación) (Pozo et al., 2008). Este proceso también es efectivo para desarrollar el sentido de su identidad como aprendiz y como intérprete. El sentido de auto percepción influiría de una manera significativa en las creencias de los alumnos sobre sus habilidades, estrategias de práctica, y en cómo el alumno va a actuar en sucesivos conciertos o audiciones (Hewitt, 2009).

A continuación, analizamos los aspectos referidos en el currículo de música acerca de las distintas dimensiones del aprendizaje de instrumento citadas: ¿cómo se evalúa?, ¿qué se evalúa?, ¿qué referente se utiliza? y ¿para qué se evalúa? 


\section{Aspectos contenidos en el currículo de música acerca de los procedimientos de evaluación}

Sobre el cómo evaluar, el currículo deja abierto a los departamentos y a los profesores su concreción a nivel de centro y de aula, al igual que el resto de las cuestiones metodológicas (Orden 1031, 2008). Las únicas indicaciones que aparecen en el currículo referidas a esta dimensión se centran específicamente en los puntos de referencia con los que establecer mediciones, es decir, sobre qué referente se utiliza.

El Decreto 30 (2007) dispone que los parámetros de referencia que se establezcan para medir los aprendizajes sean con respecto a los criterios de evaluación, el nivel de consecución de los objetivos generales y específicos de cada grado, y la propia evolución del alumno. En otras palabras, se propone que las valoraciones se realicen con respecto a parámetros de referencia que indiquen una evolución: cómo es con respecto a cómo era y a cómo debería ser (Zabalza, 1987). De este modo, una de las funciones del profesor sería regular y adaptar los objetivos y criterios propuestos desde el currículo y programaciones de departamento al nivel de los alumnos, teniendo en cuentas sus características y momento de desarrollo musical e instrumental (Real Decreto 1577, 2006).

Respecto al qué evaluar, o el objeto de la evaluación, la idea que prima en el Real Decreto 1577 (2006) es que se evalúe el grado en que se desarrollan las capacidades de expresión y comunicación musical de los alumnos. El medio por el que se propone este desarrollo es a través de la interpretación del repertorio de diferentes épocas y estilos, con sensibilidad estética. También se busca que el alumno amplíe el autocontrol y el conocimiento de sus propias posibilidades como intérprete, los cuales revertirán en su capacidad comunicativa y control de la situación escénica (Decreto 30, 2007).

Con relación al para qué evaluar, el currículo propone que se pueden realizar distintos tipos de evaluación según su finalidad. La evaluación sumativa tiene el fin de valorar los resultados del aprendizaje de los alumnos en cuanto al logro de los objetivos curriculares. El resultado del aprendizaje del alumno se puede considerar como un producto acabado (sumativa acreditativa), o como parte del proceso de aprendizaje, cuya estimación serviría para actuaciones pedagógicas posteriores (sumativa) (Coll et al., 2001; Hallam, 2001). Como recoge el Real Decreto 1577 (2006) del currículo de las enseñanzas profesionales, la finalidad formativa tiene la intención principal de mejorar la enseñanza y el aprendizaje: "la evaluación se constituye en una función formativa y, además, en una fuente de información sobre el mismo proceso de enseñanza convirtiéndose así en un referente fundamental de todo el proceso de enseñanza-aprendizaje" (p. 2853). Atendiendo a la finalidad formadora, el profesor se encargará de fomentar la autorregulación y el autoconocimiento del alumno en la conciencia y el desarrollo de sus capacidades (Orden 1031, 2008). En la citada orden, se establece que el profesor utilice estrategias que involucren al alumno como agente activo en su propio proceso de evaluación a fin de que el alumno participe, desde su autonomía como aprendiz, en el proceso de la construcción de su "personalidad artística" (Decreto 30, 2007, p. 58).

\section{Investigaciones sobre los procedimientos de evaluación de las interpretaciones musicales de los alumnos}

Acerca de cómo se evalúa en el contexto educativo español, Jornet, Sánchez-Delgado y González-Such (2015) y Vilar (2001) afirman que los procedimientos de evaluación no están (c) Psy, Soc, \& Educ, 2018, Vol. 10(1) 
sistematizados de forma generalizada en los conservatorios. Señalan que los profesores evalúan de una forma improvisada, lo que hace que la evaluación no tenga ni fiabilidad ni validez.

Jornet et al. (2015) llevan a cabo una de las aportaciones más recientes y pioneras con respecto al uso de distintos procedimientos para evaluar las interpretaciones de los alumnos en contextos de evaluación continua. Estos procedimientos se pueden utilizar para valorar al alumno durante todo el ciclo evaluador. Proponen el uso de unos portafolios de proceso y de producto, donde se recogen las tareas de los alumnos de forma cronológica para hacer un seguimiento de las capacidades que los alumnos van desarrollando. Se pueden utilizar para valorar tanto el desarrollo de los alumnos de forma individual durante su proceso de aprendizaje, como el nivel al que deberían llegar al final de cada grado por especialidades instrumentales. En la propuesta sobre el uso de rúbricas de Gil, Valero, Mingot y de Dios (2015), los miembros de varios departamentos instrumentales elaboraron una serie de criterios con diferentes niveles de consecución para evaluar al final de cada grado, con el fin de unificar los niveles por curso. Esta rúbrica también podría servir a los profesores como referencia del nivel que tienen que alcanzar sus alumnos durante las clases. Las rúbricas estarían insertas en los portafolios. Otros estudios realizados en España se enfocan específicamente en analizar las ideas que tienen los profesores sobre la evaluación como práctica inserta en las actividades de enseñanza y aprendizaje, y con unas finalidades continua, formativa y formadora (p.ej., Bautista et al., 2010; Bautista et al., 2011; López-Íñiguez, Pozo, \& de Dios, 2014; Torrado \& Pozo, 2008).

En la literatura anglosajona, existen una gran cantidad de estudios que se centran en analizar la forma en que los profesores evalúan en contextos de evaluación final, y con una finalidad sumativa (o acreditativa) (Cantwell \& Jeanneret, 2004; Daniel, 2001; Maugars, 2006; Saunders \& Holahan, 1997; Stanley, Brooker, \& Gilbert, 2002; Winter, 1993). Estos estudios aportan diferentes perspectivas en la búsqueda de una mayor calidad (fiabilidad y validez) y mejora en los procedimientos de evaluación de las interpretaciones instrumentales.

Por otra parte, en la literatura internacional existe un gran debate acerca de si la evaluación tiene más calidad - fiabilidad y validez - al evaluar 'del todo a las partes' (holística) o 'de las partes al todo' (criterial) en contextos de evaluación final (Fautley, 2010). Mills (1991) defiende que el evaluar una interpretación simplemente con una impresión general es la forma más parecida que hay a evaluar en contextos naturales. Partiendo de esta impresión general, el evaluador entraría posteriormente a valorar los aspectos particulares de la interpretación.

Sin embargo, hay muchos autores que proponen la evaluación en cuanto a criterios, partiendo de una valoración por parámetros específicos que se pueden ponderar para llegar a una calificación final (Saunders \& Holahan, 1997; Stanley et al., 2002; Winter, 1993). Por ejemplo, los miembros del jurado en el estudio de Saunders y Holahan (1997) valoraban el nivel de calidad y el carácter de las interpretaciones de los alumnos basándose solamente en su juicio personal. Consecuentemente, aportaban poco feedback de diagnosis a los alumnos. Los investigadores propusieron a los miembros del jurado el uso de escalas de calificación con criterios específicos. Los autores demostraron que las escalas pueden utilizase para evaluar a los alumnos con una fiabilidad sustancial. Además, podían servir para que los profesores proporcionasen una información más detallada a los alumnos sobre su rendimiento en aspectos concretos de la interpretación. La calificación global de una interpretación dependería del nivel 
alcanzado en cinco áreas particulares: la afinación, la técnica/articulación, la precisión rítmica, la interpretación y lectura a primera vista.

Respecto al qué se evalúa, Cantwell y Jeanneret (2004) consideran que la evaluación musical se debería centrar en medir la estructura del pensamiento musical profundo del alumno. Argumentan que la evaluación por criterios no garantiza la posibilidad de acceder a la forma en que el alumno analiza, comprende y construye sus interpretaciones. En la misma línea, Johnson (1997) afirma que la evaluación en términos estéticos constituye el mejor medio que tenemos de acceso al mundo experiencial de la interpretación musical. Según este autor, los sistemas de criterios deben diseñarse como un complemento a la respuesta subjetiva del examinador, y no para sustituir su juicio crítico. En ese sentido, Wrigley y Emmerson (2013) afirman que la evaluación criterial puede ayudar a aportar feedback a los alumnos, pero sólo si se puede acceder a valorar la complejidad estética de la interpretación de una manera holística.

Respecto al qué referente se utiliza, existen numerosos estudios centrados en el diferente uso con el que se articulan los criterios como referente en la evaluación de interpretaciones musicales (Mills, 1991; Stanley et al., 2002; Winter, 1993). Entendiendo la evaluación inserta en la práctica docente durante todo el continuo pedagógico, Torrado y Pozo (2008) identificaron tres concepciones de sofisticación creciente en los profesores (directa, interpretativa y constructiva) que vinculan el aprendizaje con su evaluación. En la concepción directa, la evaluación sería un juicio sobre el grado en el que el alumno haya conseguido los objetivos propuestos por el profesor. En la concepción interpretativa, la evaluación tendría una función de naturaleza correctiva. El profesor no sólo valora los resultados, sino que gestiona externamente los procesos de aprendizaje de los alumnos. Finalmente, la concepción constructiva considera la evaluación como parte intrincada en el aprendizaje. El profesor promueve la autorregulación del propio aprendizaje del alumno, por lo que el papel del alumno sería activo (en contraposición a las otras dos teorías, en las que el alumno juega un rol pasivo).

Acerca del para qué se evalúa, los estudios existentes analizan las ideas de los profesores dentro de un continuo acreditativo-pedagógico. Maugars (2006) analizó la actitud de los profesores de conservatorio hacia los exámenes de instrumento. Debido a que los exámenes en Francia están estructurados de una forma final y normativa, la autora concluyó que los profesores de su estudio entendían lo mismo por evaluar que por examinar.

En un estudio sobre concepciones de profesores de piano sobre la evaluación, Bautista et al. (2011) analizaron también la dimensión para qué. El estudio muestra que en la concepción directa, el profesor evalúa para controlar los estados de conocimiento del alumno de forma externa, supervisando la consecución de los resultados del aprendizaje. En la concepción interpretativa, el profesor también regula de una forma externa los estados de conocimiento del aprendiz, pero evalúa para supervisar la evolución de los procesos de aprendizaje y para corregir externamente los errores del alumno. En la concepción constructiva, el profesor evalúa para ajustar de forma continua las acciones de enseñanza al progreso del aprendizaje, integrando las distintas finalidades pedagógicas formativa y formadora.

Por su parte, Shuler (2011) defiende que la forma de promover la autorregulación de los alumnos (finalidad formadora) es a través de fomentar su asimilación de los criterios de evaluación. Dentro de los cuatro niveles que plantea sobre el uso de criterios para la autoevaluación de los alumnos, el nivel más profundo sería aquel donde el alumno puede 
desarrollar y aplicar autónomamente los criterios apropiados para mejorar su propia interpretación y aprendizaje.

La cesión del control por parte del profesor al alumno es una clave fundamental en su desarrollo (Torrado \& Pozo, 2008). Para poder llevar a cabo la finalidad formadora, el profesor debe promover la autorregulación y autoevaluación del alumno de su proceso de aprendizaje (Shively, 2015). En la búsqueda de una mayor profundidad en la evaluación de las propias capacidades del alumno, Daniel (2001) propuso a los alumnos un modelo de autoevaluación enfocado a que consiguieran una mayor independencia en la evaluación de sus propias interpretaciones. Según su punto de vista, el énfasis tradicional de los evaluadores ha estado en centrarse en el aspecto externo de la evaluación. Afirma que los alumnos reflexionan muy poco sobre su práctica, forma de tocar, manera de presentarse al público y desarrollo de sus habilidades como intérpretes. Daniel (2001) argumenta que la autoevaluación insta a los alumnos a comprometerse en unas evaluaciones mucho más estructuradas de sus propias capacidades, siendo un proceso que favorece una autopercepción más fundamentada. Se pregunta hasta qué punto los profesores pueden asumir el que los alumnos desarrollen una forma autónoma de autoevaluarse.

\section{El rol de los departamentos en la elaboración de los procedimientos de evaluación}

Gracias al grado de autonomía pedagógica que poseen los conservatorios en España (LOE, 2006), se espera que los departamentos de instrumento establezcan sus propios procedimientos de evaluación para el aprendizaje (Orden 1031, 2008): “Los departamentos didácticos establecerán en la programación didáctica los procedimientos para evaluar las habilidades artísticas y técnicas de los alumno" (p. 7). La citada orden también especifica que los profesores de cada departamento deben llegar a un consenso sobre los criterios con los que medir el aprendizaje musical de sus alumnos para utilizarlos como referente dentro de cada curso, además de comunicárselos a sus alumnos.

La intención es que los procedimientos y criterios de evaluación sean homogéneos dentro del departamento, independientemente del profesor que imparta docencia. En la evaluación del examen final de cada grado, el profesor participa como miembro del tribunal evaluador junto con otros compañeros de su departamento (Decreto 30, 2007). Esta medida posibilita el que los alumnos sean evaluados bajo los criterios de diferentes profesores, no solamente de su profesor-tutor de instrumento. Además favorece el que los niveles entre los alumnos del mismo curso y de distintos profesores sean similares.

El interés en que los procedimientos de evaluación promuevan el aprendizaje es algo que no sólo está plasmado en el currículo, sino que está empezando a manifestarse en la reciente investigación a nivel nacional (Gil et al., 2015; Jornet et al., 2015). En diferentes contextos académicos de educación musical, varios autores han estudiado la forma en que los miembros de un mismo departamento establecen los procedimientos de evaluación. Por ejemplo, Daniel (2001), Gabrielsson (2003) y Winter (1993) han analizado si los profesores están de acuerdo en la forma en que califican, encontrando que las ideas de desacuerdo tienden a ser las más frecuentes. Daniel (2001) expone que, en contextos de evaluación final en universidades australianas, la mayor parte de los miembros de un jurado reconocen que raramente llegan a un resultado de forma unánime o fácilmente. Gabrielsson (2003), en su 
extensiva revisión de la literatura, llegó a la conclusión de que "casi no hay criterios de acuerdo en lo que se debería juzgar, o en cómo deberían hacerse los juicios. Parece ser que los evaluadores no son conscientes de los criterios que utilizan en sus evaluaciones" (p. 255). Finalmente, Winter (1993) afirma que, incluso si se utilizaran criterios apropiados, existen otros factores contextuales que afectan al proceso evaluador como son la formación de los examinadores, su experiencia docente, así como la preparación de los alumnos.

\section{Objetivos}

El objetivo de este estudio fue investigar las ideas del profesorado de conservatorios acerca de los procedimientos de evaluación. Concretamente nos propusimos analizar: 1) La forma en que los profesores dicen que evalúan a sus alumnos clase a clase; 2) La forma en que los profesores dicen que evalúan a sus alumnos en los exámenes; y 3) Las ideas sobre el grado de acuerdo o desacuerdo en la forma en que evalúan sus compañeros de departamento.

El presente estudio no plantea ni propone ningún procedimiento de evaluación 'óptimo'. El trabajo se enmarca en la investigación descriptiva sobre las ideas que los profesores tienen acerca de distintos aspectos de la enseñanza y el aprendizaje de la interpretación (Bautista et al., 2011; Pozo et al., 2008; Torrado \& Pozo, 2008). En concreto, se enfoca en las ideas que los profesores tienen sobre la dimensión evaluadora de la enseñanza. Consideramos que las ideas de los profesores de instrumento sobre los procedimientos de evaluación es un objeto de estudio relevante en sí mismo, no sólo por su potencial influencia sobre las prácticas docentes (Torrado \& Pozo, 2008), sino también por reflejar niveles de conciencia e influencia sociocultural más elevados (Bautista, 2009; Brown, 2004).

\section{Método}

\section{Participantes}

Los participantes fueron 18 profesores de diferentes conservatorios de la Comunidad de Madrid (13 varones, 5 mujeres), con una edad entre los 34 y los 62 años. Su experiencia docente comprendía entre los 7 y los 26 años, con una media de 17.2 años y una desviación típica de 7.15 años. Todos los profesores eran titulados superiores de conservatorio y funcionarios del estado por oposición. El grupo de participantes pertenecía a tres especialidades instrumentales (Piano, Viento-Madera y Cuerda) y a los dos niveles educativos que se imparten en los conservatorios profesionales de la Comunidad de Madrid (enseñanzas elementales y profesionales), con seis profesores en cada combinación.

\section{Materiales y procedimiento}

Se elaboró un protocolo de entrevista semi-estructurada con varias preguntas abiertas, en base a los trabajos de investigación sobre la evaluación de las interpretaciones musicales en diferentes entornos académicos, aplicándolo al contexto de los conservatorios en España (Stanley et al., 2002).

La entrevista contenía tres bloques centrados en las ideas de los profesores de instrumento sobre: 1) Funciones y finalidades de la evaluación; 2) Procedimientos de 
evaluación; y 3) Procedimientos de calificación. En el presente estudio, se analizan las preguntas contenidas en el segundo bloque, que concretamente fueron:

- ¿Cómo evalúas tú a tus alumnos durante las clases?

- ¿Cómo evalúas tú a tus alumnos en los exámenes?

- ¿Crees que la forma en que tú evalúas se parece a la forma en que valúan otros profesores de tu departamento?, ¿en qué aspectos estás de acuerdo o en desacuerdo?

Se pidió a los participantes que respondieran a las diferentes preguntas de manera intuitiva y pensando en su propia práctica docente. Su duración fue de una hora aproximadamente y fueron grabadas en audio para su posterior transcripción. Se realizaron en el conservatorio donde cada profesor impartía docencia, en espacios de tiempo fuera de su jornada lectiva. Los participantes dieron su consentimiento explícito para participar en la investigación.

\section{Análisis de datos}

Las respuestas se analizaron mediante análisis de contenido (Creswell, 2005). Nos basamos en la teoría fundamentada, la cual se ha demostrado válida para analizar las ideas que tienen los profesores con respecto a distintos aspectos de la evaluación de la interpretación musical en otros contextos académicos (Gabrielsson, 2003; Wrigley \& Emmerson, 2013). Concretamente, según Côté, Salmela, Baria y Russell (1993), la teoría fundamentada nos proporciona un "conocimiento experiencial que nos permite saber la forma en que las personas perciben, crean e interpretan su mundo" (p. 127).

Confeccionamos tres sistemas de categorías, uno para cada una de nuestras preguntas de investigación. Pese a que únicamente preguntamos a los profesores sobre cómo evaluaban en las clases y en los exámenes, sus respuestas incluyeron alusiones a aspectos pertenecientes a otras dimensiones de la evaluación (qué evaluar, respecto a qué se evalúa, para qué se evalúa). De este modo, con el fin de no perder la información contenida en sus respuestas, nuestros sistemas de categorías fueron confeccionados de forma inductiva, de acuerdo a las dimensiones tratadas en la literatura previa sobre la evaluación (Bautista et al., 2011; Zabalza, 1987). En concreto, las dimensiones consideradas en el estudio fueron cómo se evalúa, qué se evalúa, qué referente se utiliza y para qué se evalúa.

Para analizar las respuestas de los profesores a la preguntas primera y segunda, utilizamos categorías de naturaleza no mutuamente excluyente, pues cada profesor pudo hacer alusión a un número ilimitado de ideas. En el análisis de la tercera pregunta, diseñamos un sistema de categorías en base a los grados de acuerdo ('Sí', 'No', 'Parcialmente' o 'No sabe'). Este sistema fue de categorías mutuamente excluyentes (p.ej., los profesores 'Sí' estaban de acuerdo o 'No' lo estaban).

Dos jueces independientes, expertos en evaluación musical, codificaron el $27.8 \%$ del total de los datos (las respuestas de cinco profesores). Calculamos el grado de acuerdo interjueces (coeficiente Kappa) y, en los tres sistemas de categorías analizados, los resultados alcanzaron valores satisfactorios (oscilando entre $0.75 \mathrm{y} 1$ ). Tras ello, la primera autora codificó las respuestas de todos los profesores. 


\section{Resultados}

El presente apartado consta de tres secciones, en las que analizamos: 1) Los procedimientos de evaluación en la evaluación clase a clase (evaluación procesual); 2) Los procedimientos de evaluación en la evaluación en los exámenes (evaluación final); y 3) Las ideas sobre el grado de acuerdo con el resto de compañeros del departamento en la forma en que evalúan. Presentamos las categorías dentro de cada dimensión en función de la frecuencia con la que aparecieron en las respuestas, de mayor a menor. No encontramos diferencias estadísticamente significativas al comparar las respuestas de los profesores respecto al grupo instrumental al que pertenecían (Piano, Viento-Madera y Cuerda). Por ello, los resultados se presentan agregados, sin establecer distinciones por grupo instrumental. En la exposición de las respuestas de los profesores, se presentan citas representativas de cada categoría para ilustrar su contenido.

\section{Ideas de los profesores acerca de los procedimientos de evaluación durante las clases}

Las dimensiones con las que articulamos nuestro análisis se centran en la forma en que se evalúa (cómo), el objeto de la evaluación (qué se evalúa), los aspectos que los profesores utilizaron para establecer mediciones (qué referente se utiliza) y la finalidad de la evaluación (para qué se evalúa). En la Tabla 1, se muestran las categorías que diseñamos para codificar las respuestas de los profesores. A continuación, presentamos un análisis descriptivo de las ideas de los profesores, organizado de mayor a menor frecuencia de aparición.

\section{Cómo se evalúa}

Nuestro foco de análisis se centró en lo que el profesor describió que realizaba para evaluar a los alumnos. Todos los 18 profesores entrevistados se refirieron a evaluar a través de la 'Observación/audición' del alumno mientras interpreta (p.ej., En el instrumento la evaluación más importante y la más habitual es por observación directa o por audición directa, es decir, mientras los alumnos van tocando, yo me voy dando cuenta de lo que han mejorado). Ocho profesores expusieron que 'Apuntan en cuadernos de clase y partituras' los aspectos que estén trabajando con el alumno en ese momento (p.ej., Tengo un cuaderno donde apunto las tareas semanales y tomo notas de lo que han hecho, si han estudiado, si no, los errores que tienen, cómo va desarrollándose la técnica y todo esto). Surgieron muy pocas ideas acerca de la forma en que los profesores evalúan, lo cual indica la dificultad de los profesores para verbalizar y explicitar procedimientos de evaluación más elaborados.

Qué se evalúa

Analizamos el contenido y/o capacidades específicas del aprendizaje de la interpretación musical en que los profesores enfocaron sus respuestas respecto a la evaluación de los alumnos durante las clases. Trece profesores se refirieron a evaluar las tareas y/o trabajo de los alumnos clase a clase. Esta categoría se denominó 'Objetivos (inespecíficos)', ya que los profesores no concretaron ni la naturaleza, ni el nivel determinado en la consecución de estos aspectos (p.ej., Siempre procuro que en cada clase [los alumnos] tengan objetivos claros, y con respecto a esos objetivos, ver si los han cumplido o no). Es decir, no especificaron a qué contenidos o capacidades se referían en concreto. Al describir las 'Estrategias de Estudio' que los alumnos 
emplearon en su dedicación al instrumento en casa, siete profesores mencionaron el observar cómo el alumno gestionaba su tiempo de estudio semanalmente, y el rendimiento que obtenía. Por ejemplo:

Que se vea también una manera de estudiar que sea provechosa para el alumno, que lo haga con inteligencia. Sobre todo que traiga las cosas y se vaya viendo que hay un avance en lo que hacemos.

De los cuatro profesores que hablaron sobre la 'Técnica', un profesor priorizó el desarrollo de las habilidades técnicas - entendidas como las gestión de las habilidades motrices - a las interpretativas en este nivel de aprendizaje (p.ej., Yo pienso que en Grado Elemental y Medio tenemos que darle toda la técnica posible para que luego desarrolle su capacidad musical. Yo, sobre todo, baso mi evaluación en cumplir los objetivos a nivel técnico). Respecto a la 'Calidad de la ejecución del repertorio', tres profesores se refirieron a la forma en que los alumnos tocaban las obras, y los aspectos en los que pudieran haber mejorado o no de una clase a otra, pero tampoco concretaron estos aspectos (p.ej., Intento ver qué cambios ha habido con respecto a las semanas anteriores o a la semana anterior, en los aspectos u obras que se estén trabajando). No encontramos ninguna respuesta cuyo foco fuera la 'Interpretación'. Tres profesores expusieron la idea de evaluar el 'Progreso en el aprendizaje' de una forma global y a largo plazo, valorando el desarrollo integral del alumno como intérprete (p.ej., A pesar de saber que es una enseñanza a largo plazo, donde el aprendizaje es lento, evalúo que haya un progreso).

Además de estas cinco ideas, identificamos otras tres que aparecieron con menor frecuencia. Dos profesores resaltaron la 'Actitud' favorable al estudio de la interpretación (p.ej., Voy evaluando todo en general, su actitud, su trabajo, su continuidad, los problemas que pueda tener en un aspecto u otro en ese sentido). Solamente un profesor se refirió a la 'Expresividad' - cómo el alumno comunica, desde la subjetividad, su visión personal de las obras de repertorio - a la hora de valorar musicalmente a un alumno en clase (p.ej., También valoras si van desarrollándose de manera expresiva con el trabajo. Y puede pasar en la misma clase). Por último, otro profesor aludió a las obras que el alumno pueda tocar de 'Memoria' sin la partitura - (p.ej., Yo siempre tengo un cuaderno en clase y apunto dos cosas, primero, el programa que tocan en cada clase y cómo lo tocan, si van a tocar de memoria o no).

Qué referente se utiliza

Nos centramos en los aspectos que los profesores consideraron al establecer los puntos de referencia con los que medir la evolución de los alumnos. Quince profesores explicaron que evalúan comparando el 'Nivel del alumno' con respecto a sí mismo y el desarrollo de sus capacidades (p.ej., Voy evaluando individualmente los problemas que cada uno tiene). Nueve profesores indicaron que toman como referente el 'Nivel y/o objetivos (inespecífico) propuestos por profesor' (p.ej., Tú estableces unas tareas para ciertos trabajos y evalúas si esos pasos, esas tareas, se han conseguido o no).

Para qué se evalúa

Analizamos la finalidad para la que los profesores dijeron que evalúan en el continuo acreditativo-pedagógico. Trece profesores se refirieron a 'Supervisar la evolución de los resultados de aprendizaje' de una clase para la siguiente (p.ej., La semana siguiente vamos 
viendo lo que teníamos que mejorar de la clase anterior, si está bien ponemos un tick de bien y si está mal, ponemos el tick rojo). Doce profesores mencionaron el 'Ajustar de forma continua las acciones de enseñanza al progreso del aprendizaje'. Un profesor afirmó que va modificando su práctica evaluadora dependiendo de las características del alumno (p.ej., A nivel de profesor, no tengo un tipo de evaluación establecida. Voy adaptándola en función de los problemas de cada alumno y de lo que creo que cada alumno necesita). Otro profesor explicó que en una misma clase flexibiliza los objetivos de aprendizaje según vaya respondiendo el alumno (p.ej., En mis clases con los pequeños hay mucha improvisación en el sentido de que, sobre la marcha, vemos qué funciona y qué no funciona. Entonces, se te ocurre cambiarle, darle otra cosita, insistir sobre algo o volver hacia atrás).

Nueve profesores se refirieron a 'Supervisar la evolución de los procesos de aprendizaje'. Un caso sería el del siguiente profesor, quien explicó cómo se fija en las estrategias de estudio que tiene el alumno y en su capacidad de resolver los problemas que le surgen en el estudio del repertorio en su práctica semanal (p.ej., Yo lo miro y veo qué cosas ha tenido que repetir más durante la semana porque no ha sido capaz de conseguirlas, entonces soy capaz de ver la estrategia semanal que tiene el propio alumno y dónde tiene más problemas). Cinco profesores expresaron que 'Fomentan la autonomía del alumno', principalmente a través de desarrollar su sentido crítico en la resolución de problemas (p.ej., Para fomentar su criterio ellos tienen que identificar los problemas que tienen). Otros profesores explicaron la forma en que hacen partícipe al alumno de su propio proceso de evaluación (p.ej., Les digo que toquen y luego les digo: "Cuéntame, cuéntame tú [el alumno], no yo, porque si tú no tienes nada de autocrítica, tienes muchos menos recursos para trabajar esta semana”). El profesor explicó que dialoga con el alumno y le hace comprender cómo le ha evaluado, haciéndole partícipe de su propia evaluación. Por ejemplo:

La forma en que yo evalúo es por observación directa y luego hago una especie de autoevaluación o de consenso entre los dos, porque puede ser que yo no vea lo mismo que está viendo él. Yo lo puedo tener muy claro, pero al final, la evaluación es una herramienta para ayudar al alumno a conseguir los objetivos. Si él no está entendiendo lo que yo estoy evaluando, al final, soy un juez. 
Tabla 1. Categorías a las que los profesores se refirieron en sus respuestas acerca de cómo evalúan clase a clase

\begin{tabular}{|c|c|c|c|c|c|c|c|c|c|c|c|c|c|c|c|c|c|c|c|}
\hline CLASE A CLASE & P.1 & P.2 & P.3 & P.4 & P.5 & P.6 & P.7 & P.8 & P.9 & P.10 & P.11 & P.12 & P.13 & P.14 & P.15 & P.16 & P.17 & P.18 & $\begin{array}{c}\text { Número } \\
\text { profesores }\end{array}$ \\
\hline \multicolumn{20}{|l|}{ CÓMO SE EVALUA } \\
\hline $\begin{array}{l}\text { Por observación y/o } \\
\text { audición }\end{array}$ & $\mathrm{X}$ & $\mathrm{X}$ & $\mathrm{X}$ & $\mathrm{X}$ & $\mathrm{X}$ & $\mathrm{X}$ & $\mathrm{X}$ & $\mathrm{X}$ & $\mathrm{X}$ & $\mathrm{X}$ & $\mathrm{X}$ & $\mathrm{X}$ & $X$ & $\mathrm{X}$ & $\mathrm{X}$ & $\mathrm{X}$ & $\mathrm{X}$ & $\mathrm{X}$ & 18 \\
\hline $\begin{array}{l}\text { Apuntan en cuadernos de } \\
\text { clase y partituras }\end{array}$ & X & $X$ & & $\mathrm{X}$ & & $\mathrm{X}$ & & & & $X$ & $X$ & & & $X$ & & & $X$ & & 8 \\
\hline \multicolumn{20}{|l|}{ QUÉ SE EVALÚA } \\
\hline Objetivos (inespecíficos) & $\mathrm{X}$ & $\mathrm{X}$ & $\mathrm{X}$ & $\mathrm{X}$ & $\mathrm{X}$ & $\mathrm{X}$ & & & $\mathrm{X}$ & $\mathrm{X}$ & $\mathrm{X}$ & & & $\mathrm{X}$ & $\mathrm{X}$ & & $\mathrm{X}$ & $\mathrm{X}$ & 13 \\
\hline Estrategias de estudio & & & & & & & $\mathrm{X}$ & & & $\mathrm{X}$ & & & & $\mathrm{X}$ & $\mathrm{X}$ & $\mathrm{X}$ & $\mathrm{X}$ & & 7 \\
\hline Técnica & & $\mathrm{X}$ & & & & & & & $\mathrm{X}$ & & & & & $\mathrm{X}$ & & & $\mathrm{X}$ & & 4 \\
\hline $\begin{array}{l}\text { Calidad ejecución } \\
\text { repertorio }\end{array}$ & & & & & & & $\mathrm{X}$ & & & & $\mathrm{X}$ & & & & & & $\mathrm{X}$ & & 3 \\
\hline Progreso alumno & & & $\mathrm{X}$ & & & & & & & & & & $\mathrm{X}$ & & $\mathrm{X}$ & & & & 3 \\
\hline Actitud & & & $\mathrm{X}$ & & & & & & & & & & $\mathrm{X}$ & & & & & & 2 \\
\hline Expresividad & & & & & & $\mathrm{X}$ & & & & & & & & & & & & & 1 \\
\hline Memoria & & & & & & & & & & & $\mathrm{X}$ & & & & & & & & 1 \\
\hline \multicolumn{20}{|l|}{$\begin{array}{l}\text { QUÉ REFERENTE SE } \\
\text { UTILIZA }\end{array}$} \\
\hline Nivel del alumno & $\mathrm{X}$ & $\mathrm{X}$ & & $\mathrm{X}$ & $\mathrm{X}$ & & $\mathrm{X}$ & $\mathrm{X}$ & & $\mathrm{X}$ & $\mathrm{X}$ & $\mathrm{X}$ & $\mathrm{X}$ & $\mathrm{X}$ & $\mathrm{X}$ & $\mathrm{X}$ & $\mathrm{X}$ & $\mathrm{X}$ & 15 \\
\hline $\begin{array}{l}\text { Nivel y/o objetivos } \\
\text { (inespecífico), } \\
\text { establecidos por el } \\
\text { profesor }\end{array}$ & $\mathrm{X}$ & & & $X$ & $\mathrm{X}$ & $\mathrm{X}$ & & & $\mathrm{X}$ & $\mathrm{X}$ & & $X$ & & & $X$ & & & $X$ & 9 \\
\hline \multicolumn{20}{|l|}{ PARA QUÉ SE EVALUA } \\
\hline $\begin{array}{l}\text { Supervisar la evolución } \\
\text { de los resultados de } \\
\text { aprendizaje }\end{array}$ & $X$ & $\mathrm{X}$ & & $\mathrm{X}$ & $\mathrm{X}$ & $\mathrm{X}$ & & $\mathrm{X}$ & $\mathrm{X}$ & $\mathrm{X}$ & $\mathrm{X}$ & $X$ & & $\mathrm{X}$ & & & $\mathrm{X}$ & $\mathrm{X}$ & 13 \\
\hline $\begin{array}{l}\text { Ajustar las acciones de } \\
\text { enseñanza al progreso del } \\
\text { aprendizaje }\end{array}$ & $\mathrm{X}$ & $\mathrm{X}$ & & $\mathrm{X}$ & $\mathrm{X}$ & $\mathrm{X}$ & & $\mathrm{X}$ & & $\mathrm{X}$ & $\mathrm{X}$ & $X$ & $X$ & $X$ & & & $X$ & & 12 \\
\hline $\begin{array}{l}\text { Supervisar la evolución } \\
\text { de los procesos de } \\
\text { aprendizaje }\end{array}$ & & & $X$ & & & & $X$ & & & $X$ & & $X$ & $X$ & & $X$ & $X$ & $X$ & $X$ & 9 \\
\hline $\begin{array}{l}\text { Fomentar la autonomía } \\
\text { del alumno }\end{array}$ & & & & & & & & & & $X$ & & & & & $X$ & $X$ & $X$ & $X$ & 5 \\
\hline
\end{tabular}




\section{Ideas de los profesores acerca de los procedimientos de evaluación en los exámenes}

Para analizar las respuestas de los profesores sobre la forma en que evaluaban en los exámenes, utilizamos otro sistema de categorías con las mismas cuatro dimensiones con las que examinamos las ideas de la evaluación clase a clase. Las categorías de esta segunda pregunta de investigación difieren notablemente de las categorías del primer sistema, al ser categorías que se construyeron inductivamente, pues las respuestas de los profesores fueron diferentes. En la Tabla 2, se muestran las categorías a las que cada profesor se refirió en sus respuestas.

\section{Cómo se evalúa}

Todos los profesores declararon que, al examinar, valoran principalmente por 'Observación/ audición' directa lo que hace el alumno (p.ej., Vemos cómo el niño se enfrenta a una situación que es estresante (...) Están calificándole, les están evaluando, le están observando, le están escuchando). Tres profesores expresaron que 'Se puntúa (califica)' cuando valoran de forma cuantitativa los exámenes, sin reflejar o explicitar con más detalle la forma en que pudieran haber llegado a esa calificación (p.ej., Yo, por ejemplo, sí soy partidario de hacer un examen, poner la nota del examen y que esa sea la del curso). En síntesis, los profesores solamente hicieron mención a estas dos categorías en sus respuestas a la forma en que evalúan en contextos de evaluación final.

\section{Qué se evalúa}

El aspecto al que más profesores se refirieron fue a la 'Actitud escénica' (11 participantes), como la actitud y/o control de la situación a la hora de tocar en público (p.ej., Lo único que quiero ver en esos exámenes es cuál es su capacidad de reacción ante una situación de tensión determinada que no tienen habitualmente en las clases, porque al escuchar la palabra examen aparecen los miedos). Cinco profesores articularon aspectos referidos a la 'Interpretación' del repertorio - la forma en que los alumnos entienden y dan sentido musical a las partituras a través de su instrumento - (p.ej., Partiendo de ese control [escénico], ya entras en otras cosas: estilo, pulsación, pedalización, dinámicas, fraseo, que todo sea coherente). Otros cinco expresaron que valoran la consecución de los aspectos 'Técnicos' necesarios para una interpretación fluida (p.ej., Cuando les estoy escuchando, estoy repasando en mi cabeza las cosas básicas que tengo en mente: postura, afinación, etc.). Finalmente, dos profesores mencionaron el desarrollo de la 'Memoria', en este caso como un aspecto a trabajar desde las clases (p.ej., Si yo quiero que lo que vayan a tocar en el examen, lo toquen de memoria, evidentemente ya en clase se lo he pedido de memoria).

\section{Qué referente se utiliza}

Los profesores que explicitaron algún referente para establecer mediciones se refirieron a distintas formas de articular los criterios de evaluación, y a comparar el nivel de los alumnos del mismo curso. Dos profesores explicaron que en los exámenes evalúan 'Partiendo de la impresión general hasta los criterios particulares', o del todo a las partes (p.ej., Primero, cuál es mi impresión general como músico o como intérprete de lo que está haciendo y luego ya, si quiero hacer una evaluación más fina, empiezo a intentar valorar parámetros más específicos: afinación, actitud corporal, fraseo, articulación). Dos profesores explicaron que evalúan fijándose en algún elemento o parámetro en concreto, 'Desde los criterios particulares' (p.ej., 
En un momento final. Control de la situación, control de los nervios (...), esta enseñanza es así (...) Claro, partiendo de ese control, ya entras en otras cosas: estilo, pulsación, pedalización, dinámicas, fraseo).

Un profesor mencionó la importancia de valorar el aprendizaje de los alumnos 'Siguiendo los criterios de la programación', refiriéndose a los criterios contenidos en la programación del $6^{\circ}$ curso de enseñanzas profesionales (p.ej., Obviamente ante un examen ya con tribunal ahora en $6^{\circ}$ hay que ajustarse a unos ciertos mínimos). Un profesor también expuso como referente el nivel de compañeros del mismo curso al 'Comparar el nivel con otros alumnos', para calificar a sus alumnos equitativamente (p.ej., Si en un curso concreto veo otros alumnos del conservatorio que están mejor o peor, pues yo también pongo mi nota en función de eso). A la hora de relacionarse con otros agentes en la evaluación, sólo un profesor expresó que 'Contrasta el criterio y/o nivel con otros profesores' (p.ej., En los exámenes nos juntamos con los otros "profes" de violín (...) y aunque cada uno pone la nota a sus propios alumnos, sí que tengo en cuenta las opiniones de los otros profes).

\section{Para qué se evalúa}

Trece profesores expresaron que evalúan para 'Supervisar la consecución de los resultados de aprendizaje' (p.ej., Yo, por ejemplo, sí soy partidario de hacer un examen, poner la nota del examen y que esa sea la del curso (...). Creo que tú debes evaluar lo que ves a nivel de control). Once participantes se refirieron a 'Supervisar la evolución de los procesos de aprendizaje' (p.ej., Me interesa ver [en el examen] si se refleja lo que estamos viendo en clase). Tres profesores manifestaron que 'Fomentan la autonomía del alumno' a través de compartir los criterios de evaluación con los que va a ser valorado (p.ej., En los exámenes hacemos una coevaluación, en realidad (...) vamos hablando de los diferentes criterios que se pueden tener a la hora de evaluar a cualquier músico). Un profesor explicó que implica al alumno como agente activo en su propio proceso de evaluación en los exámenes (p.ej., Si un día en una audición las cosas no funcionan (...), podemos [el profesor y el alumno] valorar por qué y qué es lo que ha podido suceder en el caso de que él en clase haya tenido una buena evolución y haya ido manejando bien la obra). 
Tabla 2. Categorías a las que los profesores se refirieron en sus respuestas acerca de cómo evalúan en los exámenes

\begin{tabular}{|c|c|c|c|c|c|c|c|c|c|c|c|c|c|c|c|c|c|c|c|}
\hline EXAMENES & P.1 & P.2 & P.3 & P.4 & P.5 & P.6 & P.7 & P.8 & P.9 & P.10 & P.11 & P.12 & P.13 & P.14 & P.15 & P.16 & P.17 & P.18 & $\begin{array}{c}\text { Número } \\
\text { profesores }\end{array}$ \\
\hline \multicolumn{20}{|l|}{ CÓMO SE EVALÚA } \\
\hline $\begin{array}{l}\text { Por observación y/o } \\
\text { audición }\end{array}$ & $X$ & $X$ & $X$ & $X$ & $X$ & $X$ & $X$ & $X$ & $\mathrm{X}$ & $X$ & $X$ & $X$ & $X$ & $X$ & $X$ & $X$ & $X$ & $X$ & 18 \\
\hline Se califica & & & & & & & & & $\mathrm{X}$ & & $\mathrm{X}$ & & & & & & $\mathrm{X}$ & & 3 \\
\hline \multicolumn{20}{|l|}{ QUÉ SE EVALÚA } \\
\hline Actitud escénica & $\mathrm{X}$ & & & $\mathrm{X}$ & & $\mathrm{X}$ & $\mathrm{X}$ & $\mathrm{X}$ & & $\mathrm{X}$ & & $\mathrm{X}$ & $\mathrm{X}$ & $\mathrm{X}$ & $\mathrm{X}$ & & & $\mathrm{X}$ & 11 \\
\hline Interpretación & $\mathrm{X}$ & & & & $\mathrm{X}$ & & & & & & & $\mathrm{X}$ & & & $\mathrm{X}$ & $\mathrm{X}$ & & & 5 \\
\hline Técnica & $\mathrm{X}$ & & & & $\mathrm{X}$ & & & & & & & $\mathrm{X}$ & & & $\mathrm{X}$ & $\mathrm{X}$ & & & 5 \\
\hline Memoria & $\mathrm{X}$ & & & & & & & & & & $\mathrm{X}$ & & & & & & & & 2 \\
\hline \multicolumn{20}{|l|}{$\begin{array}{l}\text { QUÉ REFERENTE SE } \\
\text { UTILIZA }\end{array}$} \\
\hline $\begin{array}{l}\text { Partiendo de la } \\
\text { impresión general se } \\
\text { llega a los criterios } \\
\text { particulares }\end{array}$ & $\mathrm{X}$ & & & & & & & & & & & & & & & $\mathrm{X}$ & & & 2 \\
\hline $\begin{array}{l}\text { Desde los criterios } \\
\text { particulares }\end{array}$ & & & & & & & & & & & $\mathrm{X}$ & & & & & & $\mathrm{X}$ & & 2 \\
\hline $\begin{array}{l}\text { Siguiendo los criterios } \\
\text { de la programación }\end{array}$ & & & & & & $\mathrm{X}$ & & & & & & & & & & & & & 1 \\
\hline $\begin{array}{l}\text { Contrasta el criterio y/o } \\
\text { nivel con otros } \\
\text { profesores }\end{array}$ & & & & & & & & & & & $\mathrm{X}$ & & & & & & & & 1 \\
\hline $\begin{array}{l}\text { Compara el nivel con } \\
\text { otros alumnos }\end{array}$ & & & & & & & & & & & $\mathrm{X}$ & & & & & & & & 1 \\
\hline \multicolumn{20}{|l|}{$\begin{array}{l}\text { PARA QUÉ SE } \\
\text { EVALUA }\end{array}$} \\
\hline $\begin{array}{l}\text { Supervisar la } \\
\text { consecución de los } \\
\text { resultados de } \\
\text { aprendizaje }\end{array}$ & $\mathrm{X}$ & & & $\mathrm{X}$ & $\mathrm{X}$ & $\mathrm{X}$ & & $\mathrm{X}$ & $\mathrm{X}$ & & $\mathrm{X}$ & $\mathrm{X}$ & $\mathrm{X}$ & $\mathrm{X}$ & $\mathrm{X}$ & $\mathrm{X}$ & $X$ & & 13 \\
\hline $\begin{array}{l}\text { Supervisar la evolución } \\
\text { de los procesos de } \\
\text { aprendizaje }\end{array}$ & & $X$ & $X$ & $X$ & & & $X$ & & & $X$ & $X$ & $X$ & $X$ & $X$ & & & $X$ & $X$ & 11 \\
\hline $\begin{array}{l}\text { Fomentar la autonomía } \\
\text { del alumno }\end{array}$ & & & & & & & $\mathrm{X}$ & & & & & & $X$ & & & & $X$ & & 3 \\
\hline
\end{tabular}




\section{Grado de acuerdo con los miembros del departamento}

Encontramos diferentes grados de acuerdo acerca de si los profesores suelen estar de acuerdo con sus compañeros en la forma en que evalúan. La mayor parte de los profesores indicaron 'No' estar de acuerdo con sus compañeros de departamento (12 profesores). Manifestaron sus discrepancias principalmente en la disparidad a la hora de priorizar y establecer los objetivos de aprendizaje. Concretamente, los profesores difirieron en el establecimiento de los objetivos finales que plantean a los alumnos como meta de su formación. En ese sentido, uno de los profesores argumentaba:

Yo creo que, sobre todo, estoy en desacuerdo en la disparidad en los objetivos a alcanzar (...). Para mí, dos fundamentales: uno, el de que salgan músicos, que sean músicos de verdad y no gente con una mayor o menor técnica, pero que no sean músicos $y$ luego, el segundo, es que creo que hay gente que no es realista y que cree que hay cosas que están bien, que tienen un buen nivel y que creo que la realidad y la comparativa con otros centros o cuando salen fuera te dicen que no.

Otros de los aspectos en los que expresaron estar típicamente en desacuerdo fueron en la forma en que prepararon a sus alumnos desde el trabajo de clase a los exámenes (p.ej., A veces he oído a otros profesores decir: "Bueno, pues ya para el examen [le pido al alumno que toque] de memoria" y ves que en el ensayo anterior lo están haciendo con partitura. Yo no podría hacer algo así). También en la carencia en el uso de estrategias que favorezcan la autorregulación del aprendizaje del alumno desde los criterios de evaluación (p.ej., Pues yo creo que, grosso modo, sí, es muy similar, pero cuando empieces a cambiar la escala y a aumentar el zoom, no creo que los otros compañeros hagan un seguimiento diario desde el criterio del propio alumno, sinceramente).

Tres profesores explicaron que 'No sabían' si estaban de acuerdo o no con sus compañeros del departamento, dado que nunca contrastaban su opinión con ellos al no reunirse periódicamente para realizar evaluaciones conjuntas (p.ej., Fíjate que no tengo ni idea. Es un problema. Yo, como estoy solo, no contrasto con nadie).

Las ideas de acuerdo fueron mínimas. Dos participantes coincidieron en estar 'Parcialmente' de acuerdo con sus compañeros fundamentalmente en los aspectos que consideraron más objetivos al evaluar, pero constataron que les costaba ponerse de acuerdo a la hora de valorar aspectos más subjetivos. Un ejemplo representativo de esta categoría fue:

Yo creo que tenemos claro (...), que hay determinados aspectos que serían los más objetivos de la interpretación musical que se podrían resumir en: corrección con el texto y con el estilo (...). Los aspectos más subjetivos son los que tienen que ver con el desarrollo del alumno, por ejemplo con el caso de la musicalidad, y es en lo que más diferimos.

Solamente un profesor mostró ideas en las que 'Sí' solía estar de acuerdo con la forma de evaluar de sus compañeros. En su opinión, existía una intención común de valorar a cada alumno según sus características personales, y adaptar la programación a sus necesidades de formación (p.ej., Hay una idea un poco general de intentar ayudar individualmente al alumno, (...) en el sentido de, no seguir a pies juntillas una programación porque un alumno necesita otra cosa, o no tal). 


\section{Discusión y Conclusiones}

El propósito de este estudio fue investigar las ideas del profesorado de conservatorios acerca de los procedimientos de evaluación. Concretamente nos propusimos analizar: 1) La forma en que los profesores dicen que evalúan a sus alumnos en las clases; 2) La forma en que los profesores dicen que evalúan a sus alumnos en los exámenes; y 3) Las ideas sobre el grado de acuerdo o desacuerdo en la forma en que evalúan sus compañeros. Este estudio nos permite aproximarnos a la forma en que los profesores conciben la evaluación continua, así como al grado de coherencia existente entre sus ideas sobre los procedimientos de evaluación procesual y final.

Comenzando por el cómo se evalúa, los profesores expresaron que evalúan a los alumnos y sus interpretaciones principalmente por observación y/o audición, tanto en las clases como en los exámenes. En general, los profesores controlan el desarrollo de los alumnos y hacen un seguimiento de su evolución a partir de la impresión general de lo que escuchan y ven (Mills, 1991). Pocos profesores concretaron algún procedimiento de evaluación de una manera organizada y específica (Jornet et al., 2015; Vilar, 2001). En la evaluación clase a clase, los profesores sí que mencionaron el llevar un seguimiento semanal de los aspectos trabajados con los alumnos en cuadernos y partituras, pero no describieron la estructura de estas anotaciones. En la evaluación final, muy pocos profesores se refirieron a evaluar para puntuar o calificar (Saunders \& Holahan, 1997; Stanley et al., 2002; Winter, 1993). Ningún profesor hizo mención al uso de instrumentos del tipo portafolios de producto/proceso o rúbricas similares a los propuestos por Gil et al. (2015) y Jornet et al. (2015) y que permiten sistematizar la evaluación.

En las ideas que aparecieron acerca del qué se evalúa, en la evaluación clase a clase los profesores describieron principalmente aspectos relacionados con el trabajo del repertorio. Se refirieron a secuenciar los objetivos (inespecíficos) que el alumno tiene que ir consiguiendo progresivamente en el proceso de aprendizaje durante las clases. Los profesores también mencionaron el prestar atención a las estrategias de estudio que los alumnos emplean en su estudio diario con el instrumento. En la evaluación final valoraron especialmente la presencia y actitud escénica del alumno, incluso con mayor frecuencia que las cuestiones de interpretación, técnica o memoria.

Los profesores no otorgaron gran importancia a valorar los contenidos y/o capacidades relacionados con la interpretación en sí misma (Decreto 30, 2007), ni con la forma de pensar y estructurar la música del alumno (Cantwell \& Jeanneret, 2004; Torrado \& Pozo, 2008). Prácticamente no se refirieron a fomentar en el alumno la capacidad de escucha y percepción de sus propias interpretaciones (Hallam \& Creech, 2010), al autoconocimiento de las posibilidades del alumno como intérprete (Daniel, 2001), ni al desarrollo de su expresividad musical y sus capacidades de comunicación con el instrumento (Real Decreto 1577, 2006). En otras palabras, los profesores mencionaron con poca frecuencia los aspectos interpretativos y expresivos en sí mismos, lo cual resulta paradójico pues dichos aspectos deberían ser el fundamento de la enseñanza del instrumento (Chaffin et al., 2003; Pozo et al., 2008).

Es posible que la razón por la que los profesores no mencionaran en gran medida la idea de valorar los aspectos interpretativos y expresivos se deba a que se centran en evaluar aspectos que plantean algún tipo de dificultad especial para los alumnos. Podría ser que existan aspectos que captan una mayor parte de la atención de los profesores porque tienden a plantear más problemas de aprendizaje. Por ejemplo, entendemos que los profesores identifican las estrategias de estudio y 
la actitud escénica como las capacidades que favorecerían un mejor desarrollo del alumno como instrumentista (Decreto 30, 2007). Concretamente, estos aspectos implican en sí mismos un profundo proceso de autorregulación y control del alumno en el aprendizaje de la interpretación (Torrado \& Pozo, 2008). También son aspectos que pueden favorecer o imposibilitar la interpretación en sí misma (Hallam \& Creech, 2010). Esta situación plantea una incoherencia en el ciclo evaluador (Casanova, 1995; Coll et al., 2001). Pocos profesores mencionaron el promover la autoevaluación del alumno, especialmente en estos aspectos que requieren un profundo autoconocimiento y autocontrol por parte del alumno (Hewitt, 2009), y a los que se refirieron especialmente en su forma de evaluar (Daniel, 2001; Torrado \& Pozo, 2008).

Respecto a qué referentes se utilizan para establecer mediciones y/o comparaciones, los profesores mencionaron los referentes planteados en el Decreto 30 (2007) (p.ej., criterios de evaluación, el nivel de consecución de los objetivos generales y específicos de cada grado, y la propia evolución del alumno). No obstante, articularon de forma diferente los puntos de referencia que toman en las clases y en los exámenes. En la evaluación clase a clase, los profesores mencionaron la referencia del nivel del alumno con más frecuencia de lo que mencionaron el comprobar el progreso respecto de nivel y/o objetivos (inespecíficos) propuestos por el profesor. Aproximadamente un tercio de los profesores tuvo en cuenta ambos puntos de referencia. Esto parece indicar que algunos de nuestros participantes tienen presente la finalidad formativa de la evaluación (Real Decreto 1577, 2006). En efecto, algunos profesores manifestaron ideas sobre la evaluación cercanas a las concepciones interpretativa y constructiva propuestas por Pozo et al. (2008), en las que los profesores evalúan partiendo principalmente del nivel de conocimientos y capacidades del alumno (Shuler, 2011).

En la evaluación final, la forma en que los profesores describieron el uso de los criterios fue muy heterogénea. Relacionando los resultados de nuestro estudio con estudios previos acerca de cuál es la forma más válida y fiable de evaluar, casi ningún profesor hizo mención a evaluar de una forma holística (Mills, 1991) o criterial (Gabrielsson, 2003; Saunders \& Holahan, 1997; Winter, 1993). Muy pocos profesores se refirieron a articular las interpretaciones 'del todo a las partes' o 'de las partes al todo' (Mills, 1991; Stanley et al., 2002). Análogamente, muy pocos profesores mencionaron el estructurar su percepción de las interpretaciones y/o de los aprendizajes de los alumnos con respecto a algún referente que les permitiera establecer algún tipo de mediciones o comparaciones (Shuler, 2011; Zabalza, 1987). Comparando los referentes que mencionaron en la evaluación durante las clases, los profesores tuvieron muy presente el evaluar con respecto a la propia evolución del alumno y, en menor medida, en cuanto a los objetivos de aprendizaje propuestos por él mismo. En la evaluación final, muy pocos profesores articularon algún referente y sus respuestas fueron heterogéneas.

Nuestros datos sugieren que los profesores principalmente evalúan para supervisar la evolución y consecución de los resultados de aprendizaje, ejerciendo un control externo sobre la evaluación en clases y en los exámenes. En la evaluación clase a clase, los profesores explicaron que también regulan la enseñanza al ajustar de forma continua las acciones de enseñanza al progreso del aprendizaje, con una finalidad formativa (Decreto 30, 2007). Aproximándonos al marco de las concepciones planteadas por Bautista et al. (2011) y por Torrado y Pozo (2008), podemos observar que los profesores presentaron ideas que corresponderían a perfiles mixtos. Casi ningún profesor manifestó ideas relacionadas con que evaluar es simplemente examinar, como en la concepción planteada por Maugars (2006) en el contexto de los conservatorios franceses. 
La mayor parte de los profesores mostraron posiciones en las que solamente concibieron la supervisión de los procesos y resultados del aprendizaje, así como la regulación de la enseñanza (Torrado \& Pozo, 2008). Los profesores que manifestaron posiciones más profundas para fomentar la autonomía del alumno también tuvieron ideas acerca de controlar externamente tanto los resultados como los procesos de aprendizaje de los alumnos (Bautista et al., 2011). En la evaluación final, muchos profesores se refirieron a supervisar la evolución de los procesos de aprendizaje, vinculando la evaluación clase a clase con los exámenes. De esta forma, fueron consecuentes con el concepto de evaluación continua planteado en el currículo (Decreto 30, 2007).

Muy pocos profesores se refirieron a fomentar la autonomía del alumno con una finalidad formadora a través de promover el desarrollo del criterio interpretativo propio del alumno (Daniel, 2001; Shively, 2015; Shuler, 2011). No obstante, el número de profesores que se refirieron a involucrar al alumno en su propio proceso evaluador (Orden 1031, 2008) fue mayor en la evaluación clase a clase que en los exámenes. En cierta medida, parece que algunos profesores consideraron importante implicar al alumno en su evaluación durante el proceso. Pero al hablar de unos resultados ya acabados, muy pocos profesores estimaron el ceder su control y permitir que el alumno participe activamente en su propia evaluación (Bautista et al., 2011; Fautley, 2010; Torrado \& Pozo, 2008).

Con respecto al para qué, nuestros resultados parecen indicar que la finalidad formativa de la evaluación propuesta desde la LOGSE (1990) y presente en la LOE (2006) ha calado efectivamente en las ideas de los profesores. Durante las clases, muchos profesores mencionaron el evaluar al alumno con respecto a su nivel (Torrado \& Pozo, 2008; Zabalza, 1987). También se refirieron a vincular el proceso de aprendizaje de las clases con el resultado final en los exámenes (Decreto 30, 2007). Sin embargo, en los contextos de evaluación final, los profesores no mostraron ideas que reflejaran una preocupación por evaluar de una manera sistemática y regulada, ni por establecer de una forma clara los niveles de consecución (Fautley, 2010; Jornet et al., 2015; Saunders \& Holahan, 1997; Stanley et al., 2002). Esto hace que nos cuestionemos cuáles son las "garantías para la evaluación conforme a criterios objetivos" que los profesores conciben, de acuerdo a la Orden 1031 (2008, p. 11).

Por otra parte, nuestros datos sugieren que la finalidad formadora no ha calado tanto en las ideas de los profesores. Muy pocos profesores hablaron de promover la autorregulación del alumno de su propio aprendizaje a través de la construcción conjunta de unos criterios de evaluación (Shuler, 2011), lo que podría significar que el alumno se involucre menos en su propio proceso de aprendizaje (Daniel, 2001) y en la construcción de su identidad como intérprete (Hewitt, 2009). Por lo tanto, concluimos que en las ideas de los profesores existe una clara tendencia hacia la regulación externa de los procesos de evaluación. Dichas ideas están mucho más presentes que las ideas referidas a favorecer la autoevaluación y autorregulación del alumno en su proceso de aprendizaje (Bautista et al., 2011).

En conclusión, pese a que las ideas relativas a contextos de evaluación continua y final difieren sustancialmente, ambas tienden a ser superficiales respecto a la forma de evaluar $\mathrm{y}$ fomentar los aprendizajes específicos que los alumnos han de lograr en los conservatorios, y en la finalidad para la que se evalúa.

Respecto a nuestra tercera pregunta de investigación, la mayor parte de los profesores manifestó no estar de acuerdo con la forma de evaluar de sus compañeros de departamento. Ello puede suponer el que haya una gran reticencia a la hora de establecer unos criterios y 
procedimientos de evaluación definidos y consensuados, así como a reunirse en los tribunales. Esta falta de acuerdo entre los evaluadores también se da en otros contextos académicos (Daniel, 2001; Gabrielsson, 2003).

Nuestros resultados sugieren que los profesores tienen ideas sobre la evaluación de naturaleza implícita, por lo que quizás les sea difícil articular verbalmente los procedimientos y criterios que utilizan para evaluar de una forma clara y definida (Mills, 1991; Saunders \& Holahan, 1997). Siguiendo a Fautley (2010), los profesores parecen no sentir la necesidad de utilizar unos procedimientos de evaluación más sistematizados de los que utilizan tradicionalmente, pese a estar en claro desacuerdo con los criterios de sus compañeros (Daniel, 2001; Gabrielsson, 2003). Probablemente sea difícil para los profesores profundizar en el diseño conjunto de procedimientos de evaluación si parten implícitamente de la premisa de que no van a estar de acuerdo (Daniel, 2001; Gabrielsson, 2003), y no encuentran vías para la integración de sus criterios personales (Saunders \& Holahan, 1997). Además, cada profesor parece conceptualizar la evaluación de una manera personal, no compartida y no sistematizada (Jornet et al., 2015; Vilar, 2001). Al no haber un acuerdo explícito en los procedimientos y criterios con los que evalúan, se puede correr el riesgo de que los niveles exigidos sean cada vez menos demandantes en los contextos de evaluación final. El que los niveles no estén precisados con exactitud en la evaluación final también podría tener un efecto retroactivo sobre el nivel que se exige durante las clases (Monereo, 2003).

Las principales limitaciones de nuestro estudio radican en el reducido número de profesores entrevistados y en el uso de un único procedimiento de recogida de datos (p.ej., semi-estructurada). En futuras investigaciones, además de utilizar muestras más elevadas y diversos procedimientos de recogida de información (p.ej., cuestionarios, grupos de discusión, observación), sugerimos la necesidad de centrarse en las ideas del profesorado sobre cómo evaluar aspectos más específicos (p.ej., aspectos de naturaleza técnica, de tipo interpretativa) y en analizar las relaciones entre ideas y prácticas de evaluación (Torrado \& Pozo, 2008).

\section{Implicaciones para la formación del profesorado}

Los resultados de este estudio muestran que es necesario ayudar a los profesores de conservatorio a profundizar en la explicitación de los procedimientos y criterios con los que valoran las interpretaciones de los alumnos. Ello cual contribuiría a mejorar no solo la calidad de las evaluaciones (entendida como fiabilidad y validez), sino también la calidad de la enseñanza y el aprendizaje en conservatorios a nivel más general (Hallam \& Creech, 2010; Shuler, 2011; Torrado \& Pozo, 2008). Por ejemplo, análogamente a las propuestas de Gil et al. (2015) y de Jornet et al. (2015), se podría plantear a los departamentos instrumentales que diseñaran rúbricas con criterios que contuvieran niveles de logro definidos. En el diseño de dichas rúbricas, se podrían recoger de forma oficial no solamente las notas o calificaciones de los estudiantes, sino también los objetivos, criterios y procedimientos de evaluación de una manera más detallada y estructurada (Orden 1031, 2008). Esto permitiría aportar al alumno una información cuantitativa y cualitativa sobre su proceso de aprendizaje, de forma más sistematizada.

A nuestro juicio, es imprescindible el concienciar a los profesores sobre la necesidad y los beneficios que conlleva una mayor sistematicidad en su forma de evaluar, trascendiendo la visión de la evaluación como una mera opinión que se vierte sobre el progreso de los alumnos (Jornet et 
al., 2015). Para ello sería preciso poner en marcha iniciativas de desarrollo profesional de alta calidad (Bautista, Yau, \& Wong, 2016) que permitan a los profesores aprender a diseñar procedimientos de evaluación contextualizados, mediante el trabajo colaborativo y participativo de los miembros de los departamentos instrumentales. En efecto, los propios departamentos deberían encontrar tiempos y espacios de reflexión conjunta, que faciliten el diálogo entre sus miembros (Saunders \& Holahan, 1997; Winter, 1993). Ello permitiría a los profesores articular verbalmente los procedimientos de evaluación que utilizan, de forma contextualizada, y contrastarlos con los de otros colegas para aumentar su fiabilidad y su validez (Fautley, 2010), de manera que se pueda homogeneizar todo el ciclo evaluador (Jornet et al., 2015).

Algunas posibles barreras incluyen que los profesores teman que dicha sistematización haga que sus criterios personales no se tengan en cuenta (Maugars, 2006; Winter, 1993), o que requiera un mayor empleo de tiempo en evaluar (Fautley, 2010). Argumentamos que el uso de procedimientos y criterios sistematizados reportaría notables beneficios en cuanto a la calidad de la enseñanza y el aprendizaje en conservatorios.

A nuestro juicio, es esencial que los profesores amplíen su horizonte explorando otras formas de evaluación para el aprendizaje, trascendiendo las resistencias personales y culturales del centro en el que se encuentren. La relevancia del presente estudio, por tanto, radica en documentar algunas de las diferentes perspectivas existentes entre el profesorado de los conservatorios, que podrían ayudar a otros profesores a situar sus propias ideas y a reflexionar sobre ellas. También podrían hacerles conscientes de ideas alternativas y, eventualmente, incorporar ideas de otros profesores para enriquecer o mejorar la forma en que evalúan. Este estudio también puede ayudar a los alumnos a ser conscientes de las ideas que los profesores tienen presentes al evaluar.

\section{Referencias}

Bautista, A. (2009). Concepciones de profesores y alumnos de piano sobre la enseñanza y el aprendizaje de partituras musicales. Tesis Doctoral. Universidad Autónoma de Madrid, Madrid.

Bautista, A., Pérez Echeverría, M. P., \& Pozo, J. I. (2010). Music performance teachers' conceptions about learning and instruction: a descriptive study of Spanish piano teachers. Psychology of Music, 38(1), 85-106.

Bautista, A., Pérez Echeverría, M. P., \& Pozo, J. I. (2011). Concepciones de profesores de piano sobre la evaluación. Revista de Educación, 355, 443-466.

Bautista, A., Yau, X., \& Wong, J. (2017). High-quality music teacher professional development: A review of the literature. Music Education Research, 19(4), 455-469.

Biggs, J. (1996). Enhancing teaching through constructive alignment. Higher education, 32(3), 347-364.

Brown, G. T. (2004). Teachers' conceptions of assessment: Implications for policy and professional development. Assessment in Education: Principles, Policy \& Practice, 11(3), 301-318.

Cantwell, R. H., \& Jeanneret, N. (2004). Developing a Framework for the Assessment of Musical Learning: Resolving the Dilemma of the "Parts" and the "Whole". Research Studies in Music Education, 22(1), 2-13.

Casanova, M. A. (1995). Manual de evaluación educativa. Madrid: La Muralla. 
Chaffin, R., Imreh, G., Lemieux, A. F., \& Chen, C. (2003). " Seeing the Big Picture": Piano Practice as Expert Problem Solving. Music Perception: An Interdisciplinary Journal, 20(4), 465-490.

Coll, C., Martín, E., \& Onrubia, J. (2001). La evaluación del aprendizaje escolar: dimensiones psicológicas, pedagógicas y sociales. En C. Coll, J. Palacios, \& A. Marchesi (Eds.), Desarrollo psicológico y educación, II. Psicología de la educación escolar (pp. 549-574). Madrid: Alianza Editorial.

Coll, C., \& Remesal, A. (2009). Concepciones del profesorado de matemáticas acerca de las funciones de la evaluación del aprendizaje en la educación obligatoria. Infancia y Aprendizaje, 32(3), 391-404.

Côté, J., Salmela, J. H., Baria, A., \& Russell, S. J. (1993). Organizing and interpreting unstructured qualitative data. The sport psychologist, 7(2), 127-137.

Creswell, J. W. (2005). Educational research. Planning, conducting, and evaluating quantitative and qualitative research. New Jersey: Pearson Education Inc.

Daniel, R. (2001). Self-assessment in performance. British Journal of Music Education, 18(03), 215-226.

Decreto 2618/1966, de 10 de septiembre, sobre reglamentación general de los conservatorios de música (B.O.E. núm. 254, 24 de octubre 1966).

Decreto 30/2007, de 14 de junio, del Consejo de Gobierno, por el que se establece para la Comunidad de Madrid el currículo de las enseñanzas profesionales de música (B.O.E. núm. 18,25 de junio de 2007).

Fautley, M. (2010). Assessment in music education. Oxford: Oxford University Press.

Gabrielsson, A. (2003). Music performance research at the millennium. Psychology of Music, 31(3), 221-272.

Gil, P., Valero, J. P., Mingot, B., \& de Dios, A. (2015). Elaboración de rúbricas de desempeño para portafolios de Música y Danza. En C. J. Cabedo, R., \& J. P. Valero (Eds.), Las enseñanzas de música y danza en la comunidad valenciana. Marco teórico para su evaluación desde un modelo de cohesión social (pp. 97-104). Valencia: Generalitat Valenciana.

Hallam, S. (2001). The development of metacognition in musicians: Implications for education. British Journal of Music Education, 18(01), 27-39.

Hallam, S., \& Creech, A. (2010). Issues of assessment and performance Music Education in the 21st Century in the United Kingdom: Achievements, Analysis and Aspirations. London: Institute of Education-London.

Hewitt, A. (2009). Musical Styles as Communities of Practice Challenges for learning, teaching and assessment of music in higher education. Arts and Humanities in Higher Education, 8(3), 329-337.

Ibarra, M. S., \& Rodríguez, G. (2010). Aproximación al discurso dominante sobre la evaluación del aprendizaje en la universidad. Revista de Educación 351, 385-407.

Johnson, P. (1997). Performance as Experience: the problem of assessment criteria. British Journal of Music Education, 14(03), 271-282.

Jornet, J. M., Sánchez-Delgado, P., \& González-Such, J. (2015). La evaluación de competencias musicales y de danza en conservatorios profesionales. En C. J. Cabedo, R., \& J. P. Valero (Eds.), Las enseñanzas de Música y Danza en la Comunidad Valenciana. Marco teórico para su evaluación desde la cohesión social. (pp. 81-90). Valencia: Generalitat Valenciana. 
L.O.E. (2006). Ley Orgánica 2/2006, de 3 de mayo, de Educación (BOE de 4 de mayo).

L.O.G.S.E. (1990). Ley Orgánica General del Sistema Educativo, de 3 de octubre de 1990 (BOE de 4 de octubre).

López-Íñiguez, G., Pozo, J. I., \& de Dios, M. J. (2014). The older, the wiser? Profiles of string instrument teachers with different experience according to their conceptions of teaching, learning, and evaluation. Psychology of Music, 42(2), 157-176.

Maugars, C. (2006). Attitudes of music teachers towards final examinations in the French music conservatoires. International Journal of Music Education, 24(1), 43-55.

Mills, J. (1991). Assessing musical performance musically. Educational studies, 17(2), 173-181.

Miras, M., \& Solé, I. (1990). La evaluación del aprendizaje y la evaluación en el proceso de enseñanza y aprendizaje. En C. Coll, A. Palacios, \& A. Marchesi (Eds.), Desarrollo psicológico y educación, II. Psicología de la educación escolar (pp. 419-431). Madrid: Alianza.

Monereo, C. (2003). La evaluación del conocimiento estratégico a través de tareas auténticas. Revista Pensamiento Educativo, 32, 71-89.

Orden 1031/2008, de 29 de febrero, de la Consejera de Educación, por la que se regulan para la Comunidad de Madrid la evaluación en las enseñanzas profesionales de música y los documentos de aplicación (B.O.C.M. núm. 67, de 19 de marzo de 2008).

Pozo, J. I. (1996). Aprendices y maestros. La nueva cultura del aprendizaje. Madrid: Alianza.

Pozo, J. I., Bautista, A., \& Torrado, J. A. (2008). El aprendizaje y la enseñanza de la interpretación musical: cambiando las concepciones y las prácticas. Cultura y Educación, 20(1), 5-15.

Real Decreto 1577/2006, de 22 de diciembre, por el que se fijan los aspectos básicos del currículo de las enseñanzas profesionales de música reguladas por la Ley Orgánica 2/2006, de 3 de mayo, de Educación (B.O.E. núm. 18, 20 de enero 2007).

Saunders, T. C., \& Holahan, J. M. (1997). Criteria-specific rating scales in the evaluation of high school instrumental performance. Journal of Research in Music Education, 45(2), 259-272.

Shively, J. (2015). Constructivism in Music Education. Arts Education Policy Review, 116(3), $128-136$.

Shuler, S. C. (2011). Music Assessment, Part 1: What and Why. Music Educators Journal, 98(2), $10-13$.

Stanley, M., Brooker, R., \& Gilbert, R. (2002). Examiner perceptions of using criteria in music performance assessment. Research Studies in Music Education, 18(1), 46-56.

Torrado, J. A., \& Pozo, J. I. (2008). Metas y estrategias para una práctica constructiva en la enseñanza instrumental. Cultura y Educación, 20(1), 35-48.

Vilar, J. M. (2001). La evaluación en las escuelas de música y en los conservatorios. VitoriaGasteiz: Servicio Central de Publicaciones del Gobierno.

Winter, N. (1993). Music Performance Assessment: A Study of the Effects of Training and Experience on the Criteria Used by Music Examiners. International Journal of Music Education 1, 34-39.

Wrigley, W. J., \& Emmerson, S. B. (2013). Ecological development and validation of a music performance rating scale for five instrument families. Psychology of Music, 41(1), 97-118.

Zabalza, M. Á. (1987). Diseño y desarrollo curricular. Madrid: Narcea Ediciones. 